\title{
Power Amplifiers Modeling Technique for Reconfigurable Antenna Array Application
}

\author{
G. Zakka El Nashef ${ }^{1}{ }^{*}$, F. Torres $^{1}$, S. Mons ${ }^{1}$, T. Reveyrand ${ }^{1}$, E. N'goya ${ }^{1}$, T. Monédière ${ }^{1}$, R. Quéré ${ }^{1}$ \\ ${ }^{1}$ XLIM - C2S2/OSA departments UMR CNRS n 6172 , University of Limoges 123, avenue Albert Thomas \\ - 87060 Limoges CEDEX Tel: +33 555457735 Fax: +33 555457766 e-mail: georges.zakka-el- \\ nashef@xlim.fr
}

\begin{abstract}
The design of reconfigurable active antennas requires an efficient system simulation in order to analyze the impact of other components on the array pattern, especially the Power Amplifiers (PA) effect. Therefore, the performance prediction of PA on $T x-R x$ chains is important. This paper presents a behavioral model of a PA, which is able to take into account the load impedance mismatch with a VSWR (Voltage Standing Wave Ratio) up to 3 . The proposed behavioral model, based on non-linear scattering functions, is extracted from simple CW measurements, and then implemented in Agilent Advanced Design System (ADS). Our behavioral model is validated by comparison with measurement results in the case of loading impedance mismatch (VSWR $\leq 3)$. Another model was validated (expansion of Taylor series to the second order), taking into account large VSWR (VSWR $=3.5$ ).
\end{abstract}

Index Terms - AESA, Behavioral modeling, impedance mismatch, electromagnetic interactions.

\section{INTRODUCTION}

In the last ten years, the developments of active phased array antenna devices have reached a whole new level, concerning designs, modeling and agility. Indeed, these reconfigurable antennas have large advantages such as an extremely fast scanning rate or the ability to produce multiple agile beams [1]. But the problem is that active antennas may lead to a strong load impedance mismatch (VSWR up to 3). This mismatching has a strong influence on the PA in term of gain (AMAM) and phase (AMPM). Thus, the necessary weights applied to each antenna on the array in a given pointing direction will be also modified once introduced to the PA, degrading the array efficiency and its radiation performance. Therefore, in order to obtain an accurate Tx-system, a very efficient simulation tool is needed. This paper focuses on the development of a distinct and precise PA model, in order to quantify its impact on Tx-chains. However, some load impedances used for validation were calculated using an electromagnetic macro-model representing an antenna array [2], [3]. This macro-model takes into account the strong mutual coupling between the array's antennas and allows the calculation of necessary weights (amplitudes and phases), in order to obtain an optimum radiation pattern (high gain with low side lobes levels), while taking into account the calculated load impedance $(Z L \neq 50 \Omega)$ of each antenna in the array according to the frequency and the pointing angle. Then those calculated load impedances are used instead of the real antennas to define the load impedances (Zload) of the active circuits (PAs), in order to optimize the performances of the global system.

\section{BEHAVIORAL MODEL}

\section{A. Theoretical Concept}

In the past years, several behavioral models were proposed, enabling to predict memory effects [4], [5] or thermal effects [6]. However, these models were unilateral, and dedicated to Data Flow (DF) simulators. More recently, efficient bilateral models were developed [7], using the large signal S parameter formalism based on non linear scattering functions [8]. In this paper, the behavioral model is referred to Poly-Harmonic Distorsion (PHD) behavioral model [7], but more simplified because we made the assumption that an amplifier is considered to work at the fundamental frequency without memory effects. It is defined by the following equation:

$$
\tilde{b}_{i}=f_{N L}\left\{\Re e\left(\tilde{a}_{1}\right), \mathfrak{I} m\left(\tilde{a}_{1}\right), \Re e\left(\tilde{a}_{2}\right), \mathfrak{I} m\left(\tilde{a}_{2}\right)\right\}
$$

where $\tilde{a}_{i}$ and $\tilde{b}_{i}$ are respectively the incident and reflected power waves at the two ports.

Considering $\tilde{a}_{1}$ as the reference wave and $\tilde{a}_{2}<<\tilde{a}_{1}$, the development of Taylor series limited to the first order enables us to write (1) as follows:

$$
b_{i}=S_{i 1}\left(\left|a_{1}\right|\right) \cdot a_{1}+S_{i 2}\left(\left|a_{1}\right|\right) \cdot a_{2}+T_{i 2}\left(\left|a_{1}\right|\right) \cdot a_{2}^{*}
$$

where $S_{i j}\left(\left|\tilde{a}_{1}\right|\right)$ and $T_{i 2}\left(\left|a_{1}\right|\right)$ are the nonlinear scattering functions.

We see the nonlinear mappings in this case depend only on a single real variable $\left(\left|a_{1}\right|\right)$. All the other incident wave dependences are linear. This is a dramatic simplification, and enables simple characterization and direct identification. The $\mathrm{S}$ and $\mathrm{T}$ terms allow the model to predict the component behavior for small to moderate mismatches (VSWR=3) at port 2 at the fundamental frequency.

Unfortunately, the reached boundaries concerning the VSWR were not enough, due to the problem of model accuracy working with large VSWR (VSWR=3.5). Therefore 
we expanded the bilateral model to a Taylor development of second order, and (2) becomes:

$b_{1}=S_{11} * \tilde{a}_{1}+S_{12} * \tilde{a}_{2}+S_{12}^{\Delta} * \tilde{a}_{2}^{*}+S_{12}^{\Delta \prime} * \tilde{a}_{2}^{2}+S_{12}^{\Delta \prime} * \tilde{a}_{2}^{* 2}+S_{12}^{\Delta{ }^{\prime \prime}} * \tilde{a}_{2} * \tilde{a}_{2}^{*}$ $b_{2}=S_{21} * \tilde{a}_{1}+S_{22} * \tilde{a}_{2}+S_{22}^{\Delta} * \tilde{a}_{2}^{*}+S_{22}^{\Delta^{\prime}} * \tilde{a}_{2}^{2}+S_{22}^{\Delta \prime} * \tilde{a}_{2}^{* 2}+S_{22}^{\Delta \prime^{\prime \prime}} * \tilde{a}_{2} * \tilde{a}_{2}^{*}$

\section{B. Measurement and Extraction procedures}

A measurement setup was explained in [9], which enables power amplifier characterization driven by radio frequency modulated signals (CW measurement at the operating frequency). Incident $\left(\tilde{a}_{i}\right)$ and reflected $\left(\tilde{b}_{i}\right)$ waves at both ports of the device under test can be measured in a load pull environment, using a VNA (Vectorial Network Analyzer) load-pull setup [8]. Three load impedances having an orthogonal position on Smith chart and corresponding to a low mismatching (VSWR $\leq 1.6$ ) are sufficient to solve (2) at different input powers.

In the case of second order Taylor expansion, twelve parameters in (3) instead of six need to be extracted. The extraction is carried out by measuring the four power waves with six distinct impedances in order to solve the system. The selected impedances in this case are no longer orthogonally located. The first measured impedance will be the Smith chart center and the five other impedances are selected on a constant VSWR circle with an argument of 72 degrees compared to the previous chosen one. The VSWR is between 1.3 and 1.6, and these values are chosen in order to have a large surface covered by those chosen impedances, but not large enough to make the system resolution unstable.

\section{Implementation}

The characterized device is an $8-14 \mathrm{GHz} 27 \mathrm{dBm}$ PA from NEX-TEC RF, and no electrical model is provided by the manufacturer. Extraction and validation of the PA's model have been realized in Agilent ADS in the frequency domain. The model has been implemented thanks to an FDD (Frequency-domain Defined Device) nonlinear block.

\section{NUMERICAL VALIDATION}

The fundamental AMAM and AMPM characteristics are compared in Figs. 1 and 2 respectively for the three extracted impedances.

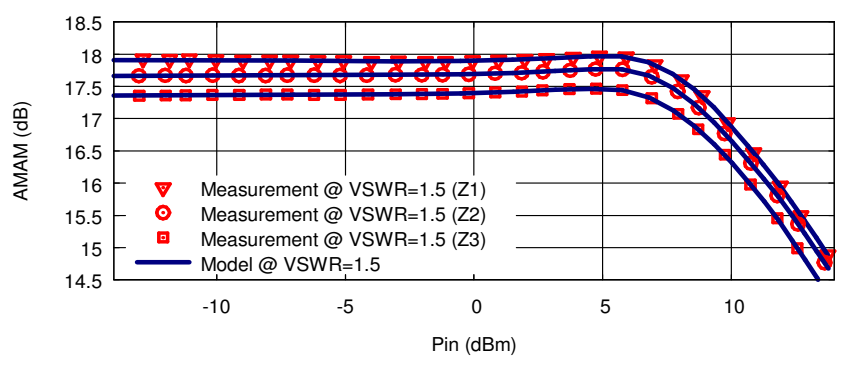

Fig. 1. Fundamental gain compression (AMAM) vs. input power for the extracted impedances $(\mathrm{Z} 1=49.1-\mathrm{j} \cdot 4.9, \quad \mathrm{Z} 2=59.8+\mathrm{j} \cdot 2.2$, $\mathrm{Z} 3=39.5+\mathrm{j} \cdot 0.2$ ). $1^{\text {st }}$ order model (lines) compared to load pull measurement (symbols).

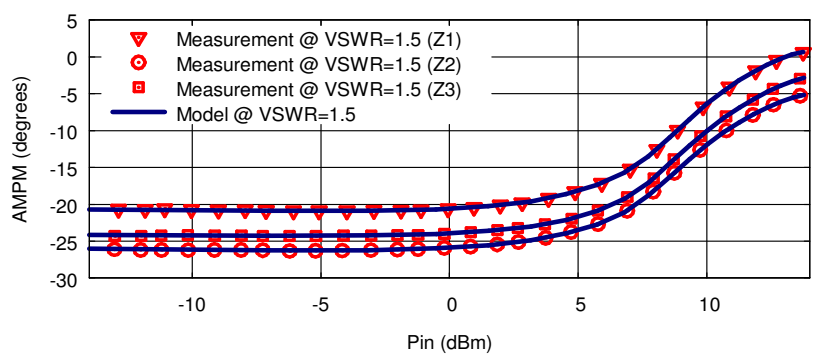

Fig. 2. Fundamental phase variation (AMPM) vs. input power for the extracted impedances $(\mathrm{Z} 1=49.1-\mathrm{j} \cdot 4.9, \quad \mathrm{Z} 2=59.8+\mathrm{j} \cdot 2.2$, $\mathrm{Z} 3=39.5+\mathrm{j} \cdot 0.2$ ). $1^{\text {st }}$ order model (lines) compared to load pull measurement (symbols).

Fig. 1 and Fig. 2 show a great agreement between the behavioral model and the measurements. Moreover other experiments for different loads on $\mathrm{VSWR}=2, \mathrm{VSWR}=3$ and VSWR $=3.5$ were performed.

Figs. 3 to 8 present the AMAM and AMPM for several chosen impedances on each VSWR, showing the prediction ability and the boundaries limitation of the PA model limited to the first order of Taylor series development.

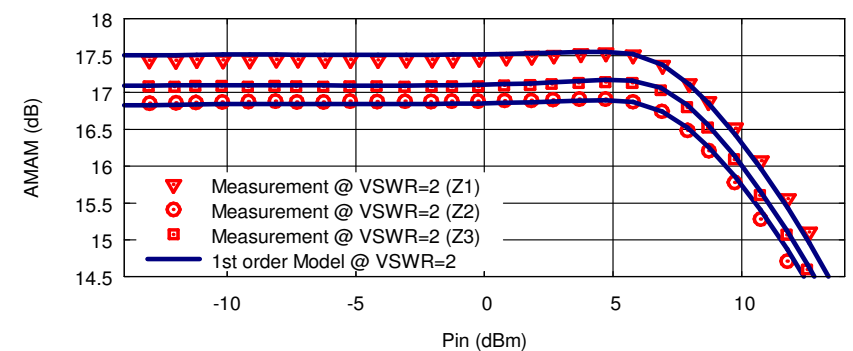

Fig. 3. Fundamental gain compression (AMAM) vs. input power for several impedances $(\mathrm{Z} 1=36.8-\mathrm{j} \cdot 27.1, \quad \mathrm{Z} 2=35.2-\mathrm{j} \cdot 5.8$, $\mathrm{Z} 3=56.3+\mathrm{j} \cdot 26.3)$ on $\mathrm{VSWR}=2$ circle. $1^{\text {st }}$ order model (lines) compared to load pull measurement (symbols).

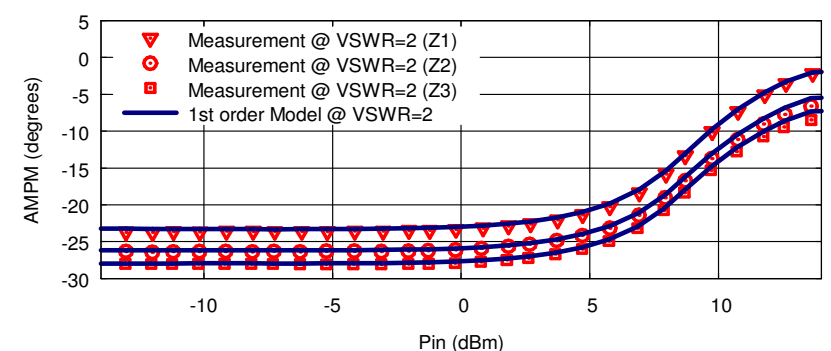

Fig. 4. Fundamental phase variation (AMPM) vs. input power for several impedances $(\mathrm{Z} 1=36.8-\mathrm{j} \cdot 27.1, \mathrm{Z} 2=35.2 \mathrm{j} \cdot \mathrm{j} .8, \mathrm{Z3}=56.3+\mathrm{j} \cdot 26.3)$ on VSWR=2 circle. $1^{\text {st }}$ order model (lines) compared to load pull measurement (symbols). 


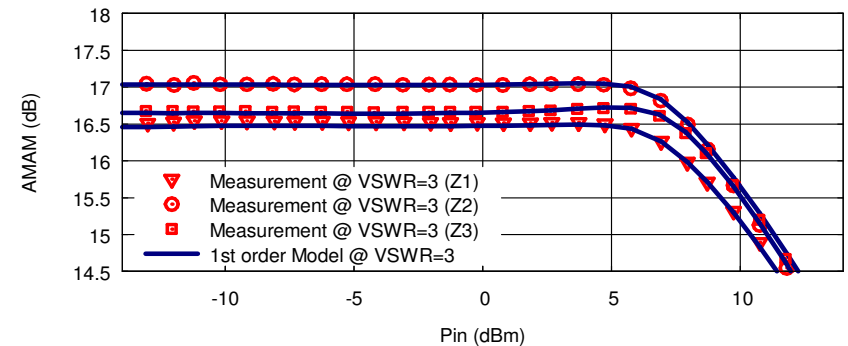

Fig. 5. Fundamental gain compression (AMAM) vs. input power for several impedances $\quad(Z 1=16.2-\mathrm{j} \cdot 0.6, \quad \mathrm{Z} 2=20.5+\mathrm{j} \cdot 24$, $\mathrm{Z} 3=111.6+\mathrm{j} \cdot 61.9)$ on $\mathrm{VSWR}=3$ circle. $1^{\mathrm{st}}$ order model (lines) compared to load pull measurement (symbols).

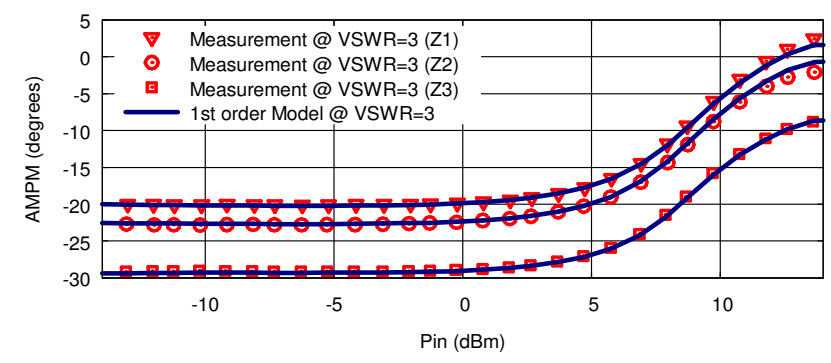

Fig. 6. Fundamental phase variation (AMPM) vs. input power for several impedances $(\mathrm{Z} 1=16.2-\mathrm{j} \cdot 0.6, \mathrm{Z} 2=20.5+\mathrm{j} \cdot 24, \mathrm{Z} 3=111.6+\mathrm{j} \cdot 61.9)$ on VSWR $=3$ circle. $1^{\text {st }}$ order model (lines) compared to load pull measurement (symbols).

Figs. 3 to 6 show a good agreement between the model and measurements, showing the prediction ability of the PA model to take into account moderate VSWR at the price of a small degradation of performances.

Moreover, we expanded the boundaries of the PA model, using some impedances on VSWR=3.5. Fig. 7 and Fig. 8 present the AMAM and AMPM for some impedances on VSWR $=3.5$, where we compare the first order model to the second order model.

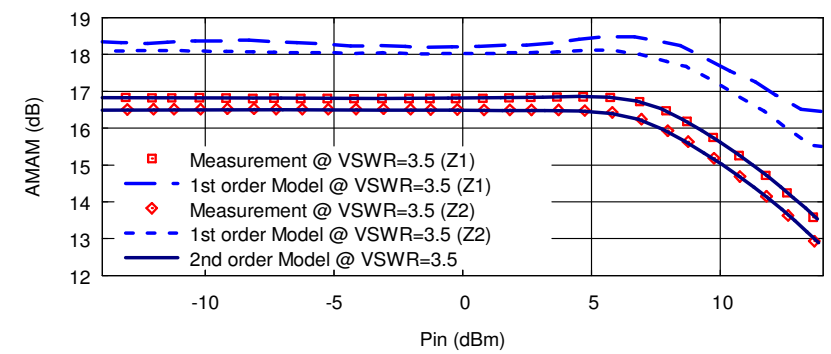

Fig. 7. Fundamental gain compression (AMAM) vs. input power for several impedances $(\mathrm{Z} 1=14.8+\mathrm{j} \cdot 10.4, \quad \mathrm{Z} 2=60.9+\mathrm{j} \cdot 67.4)$ on VSWR $=3.5$ circle. $1^{\text {st }}$ order model (blue symbols) and $2^{\text {nd }}$ order model (lines) compared to load pull measurement (red symbols).

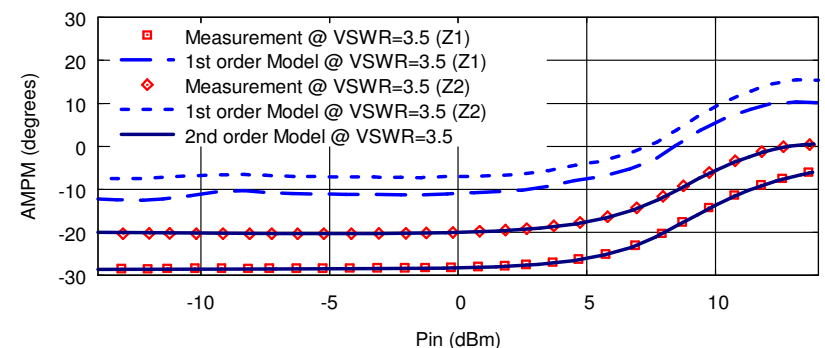

Fig. 8. Fundamental phase variation (AMPM) vs. input power for several impedances $(Z 1=14.8+\mathrm{j} \cdot 10.4, \quad \mathrm{Z} 2=60.9+\mathrm{j} \cdot 67.4) \quad$ on VSWR $=3.5$ circle. $1^{\text {st }}$ order model (blue symbols) and $2^{\text {nd }}$ order model (lines) compared to load pull measurement (red symbols).

Fig. 7 and Fig. 8 show a medium degradation between the measurement and the model concerning the $1^{\text {st }}$ order model. This factor is due to the limitation of Taylor series development to the first order. However, the second order model shows a remarkably great accuracy between the measurement and the model, indicating that the first order model is not efficient enough and the development of the Taylor series were needed, in order to improve the accuracy and to take into account large VSWR (i.e. VSWR=3.5).

On the other hand, final experiments were made using the calculated impedances obtained from the electromagnetic macro-model mentioned previously. These calculated impedances will be used instead of the real antennas to define the load impedances of the PA. Fig. 9 and Fig. 10 present the AMAM and AMPM for some calculated impedances, using the $1^{\text {st }}$ order model (VSWR obtained are less or equal to 3 ).

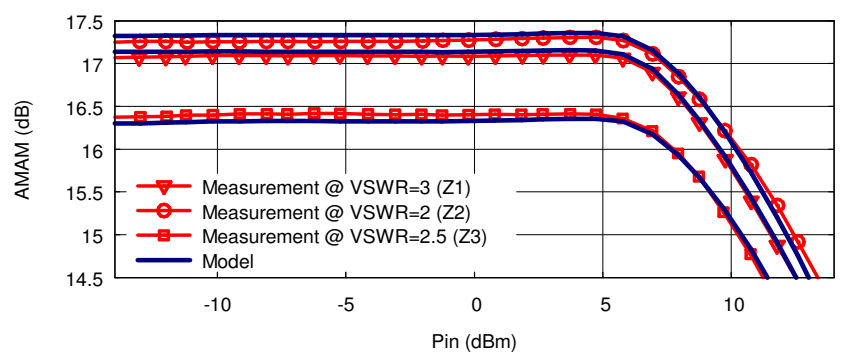

Fig. 9. Fundamental gain compression (AMAM) vs. input power for several calculated impedances $(\mathrm{Z} 1=20.6+\mathrm{j} \cdot 12.7, \mathrm{Z} 2=26.2 \mathrm{j} \cdot 0.004$, $\mathrm{Z} 3=21.6+\mathrm{j} \cdot 18.8)$ using the electromagnetic analysis. model (lines) compared to load pull measurement (symbols). 


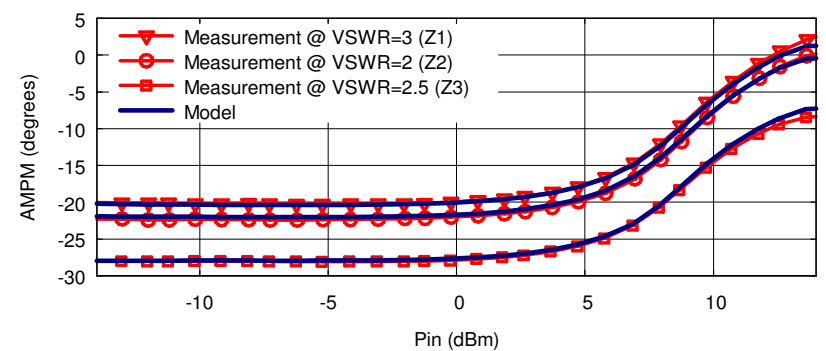

Fig. 10. Fundamental phase variation (AMPM) vs. input power for several calculated impedances $(\mathrm{Z} 1=20.6+\mathrm{j} \cdot 12.7, \mathrm{Z} 2=26.2 \mathrm{j} \cdot 0.004$, $\mathrm{Z} 3=21.6+\mathrm{j} \cdot 18.8)$ using the electromagnetic analysis. model (lines) compared to load pull measurement (symbols).

Fig. 9 and Fig. 10 show a good agreement between the model and the measurement. These results show us a first approach of coupling both simulation tools, which indicate the prediction ability of the PA model at system level with a great and accurate response.

\section{CONCLUSION}

A scattering parameter approach, based on the PHD model, has been presented. This approach takes into account mismatching effects applied to a PA. It allows a good prediction of a PA's behavior in the case of an output loading mismatch (up to VSWR=3), also useful in establishing predictive performances in agile array applications. Moreover, we have demonstrated that the first order model is not efficient enough for impedances on VSWR $\geq 3$, where we extended the Taylor series development to the second order, to improve the accuracy and take into account large VSWR (i.e. VSWR $\geq 3$ ).

Finally, this static model (operating at the fundamental frequency), implemented in $\mathrm{ADS}$, is a first approach for reconfigurable antenna application. The model's abilities could be extended to include the memory effects and the prediction of thermal effects.

\section{ACKNOWLEDGEMENT}

This work was realized within the framework of 'Lypsis project', labellized by the pole Elopsys (pole of competitiveness of high technologies in Limousin France www.elopsys.fr), and supported by the French "Direction Générale des Entreprises".

\section{REFERENCES}

[1] H. Hommel, H.P. Feldle, "Current Status of Airborne Active Phased Array (AESA) Radar System and Future Trends", 34th European Microwave Conference, Amsterdam 2004.

[2] J. Drouet, M. Thevenot, R. Chantalat, C. Menudier, M. Koubeissi, T. Monédière, and B. Jecko, "Global Synthesis Method for the Optimization of Multifeed EBG Antennas", International Journal of Antennas and Propagation, Vol. 2008, Article ID 790358.

[3] G. Zakka El Nashef, F. Torres, A. El Sayed Ahmad, T. Monédière, M. Thévenot, S. Mons, E. N'Goya, R. Quéré, "Development of an Electromagnetic Macro-model for Reconfigurable Array Application", 4th European Conference on Antennas and Propagation (EuCAP 2010), 12-16 April 2010, Barcelona, Spain.

[4] A. Soury, E. N'Goya, J.M. Nebus,"'A New Behavioral Model Taking into Account Nonlinear Memory Effects and Transcient Behaviors in Wideband SSPAs", IEEE MTT-S Int Microwave Symp. Digest, June 2002, pp. 853-856.

[5] C. Maziere, A. Soury, E. N'Goya, J.M. Nebus, “A System Level Model of Solid State Amplifiers with Memory Based on a Nonlinear Feedback Loop Principle", European Microwave Conference, Paris France 2005.

[6] S. Boumaiza, J. Gauthier, F.M. Ghannouchi," Dynamic Electrothermal Behavioral for RF Power Amplifiers," IEEE MTT-S International Microwave Symposium Digest, June 2003, pp 351-354.

[7] D.E. Root, J. Verspecht, D.sharrit, J. Wood, A. Cognata, "Broad-Band Poly-Harmonic Distorsion (PHD) Behavioral Models from Fast Automated Simulations and Large-Signal Vectorial Network Measurement", IEEE Transactions on Microwave Theory and Techniques, vol. 53, n011,pp. 36563664, Nov. 2005.

[8] J.Verspecht, "Scattering Functions for Nonlinear Behavioral Modeling in the frequency domain", Fundamentals of Nonlinear Behavioral Modeling, Foundations and Applications workshop, IEEE MTT-S, International Microwave Symposium, June 2003.

[9] T. Reveyrand, C. Mazière, J.M. Nebus, R. Quéré, A. Mallet, L. Lapierre, J. Sombrin, "A Calibrated time domain Envelope Measurement System for the Behavioral Modeling of Power Amplifier", European Microwave Conference (EUMC) 2002, Milan, GAAS, $\quad$ pp. 237-240, 2002. 